\title{
Local-scale solute transport in variously structured soils under continuous flood irrigation
}

\author{
A. Comegna ${ }^{1}$, A. Coppola ${ }^{2}$, A. Sommella ${ }^{1} \&$ G. Severino ${ }^{1}$ \\ ${ }^{1}$ Department of Agricultural Engineering and Agronomy, \\ University of Naples Federico II, Italy \\ ${ }^{2}$ DITEC Department, University of Basilicata, Potenza, Italy
}

\begin{abstract}
This paper reports the results of research carried out to establish the possibility of using Time Domain Reflectometry (TDR) technology in measuring breakthrough curves of anion tracers such as chloride and bromide. The test conditions adopted refer to the case of undisturbed porous media with bimodal porosity under saturated-unsaturated conditions and to a liquid phase of assigned chemical characteristics. The BTCs were measured with vertically installed TDR probes. Interpretation of signal impedance was carried out in a similar fashion to Kachanosky et al. so as to obtain estimates of the minimum impedance load $Z_{0}$. The measured BTCs were interpreted to estimate transport parameters by using a two-parameter convective-dispersive model. Finally, a sensitivity analysis of the hydrodynamic dispersion parameter as related both to the average pore water velocity $v$, and TDR measurement errors were performed.

Keywords: solute transport models, chloride breakthrough curve, TDR calibration.
\end{abstract}

\section{Introduction}

Contamination of groundwater has received considerable attention in recent years because of concerns about degradation of this important source of fresh water, with possible toxicological consequences. Contamination has often been associated with point sources such as disposal sites or illegal dumping of chemical and industrial wastes. More recently, non-point pollution has become the center of increased attention by the research community, both in urban and 
agricultural landscapes. Although a vast amount of complex mathematical flow and transport models exist, reliable predictions of flow and transport processes in natural, heterogeneous soils can only be made if expected spatial and temporal variability of the soil-atmosphere interface where diffuse pollution almost always occurs is also addressed. Unfortunately, the limiting step in our understanding of solute transport at field scale is just a lack of reliable and sufficient data. Indeed, at present investigations on solute transport in porous media for assessing solute leaching potential mainly base on time-consuming laboratory or field experiments (Kutilek and Nielsen, [2]; Jury et al., [3]).

Recent studies have demonstrated the potential of Time Domain Reflectometry (TDR) technology as an alternative technique to estimate chemical transport parameters in soil (Kachanosky et al., [1]; Comegna et al., [4]). This procedure could be recognized as an effective means for characterizing the solute transport behavior in soil.

Given the advantages of acquiring in real-time a suitable number of laboratory and field observations, which are particularly useful in parallel basic research, the Institute of Agricultural Hydraulics of the University of Naples "Federico II" together with DITEC of the University of Basilicata set up an equipment with a view to defining sufficiently and completely the parameters of chemical transport in soil for the widespread future use of simulation models. The main objective of this study is to illustrate the application of TDR methodology for estimating solute transport parameters from miscible experiments at laboratory scale. Main problems arising when applying the methodology to undisturbed soils with different aggregation degrees are also addressed. In the last part of the work results of modelling of experimental data are illustrated and a sensitivity analysis is also performed, mainly aiming to verify the robustness of fitted hydrodynamic dispersion parameter as related to experimental errors.

\section{Experimental methodology}

\subsection{TDR in solute transport studies}

A time domain reflectometry (TDR) waveform contains information describing both the speed of propagation and the attenuation of an electromagnetic pulse as it travels along a waveguide or probe (Topp et al., [5]; Spaans and Baker, [6]). Use of these properties allows for the determination of both the water content $\theta$ and the bulk electrical conductivity $E C_{\mathrm{a}}$ in approximately the same volume of porous medium (Dalton et al., [7]). Through long-established relationships describing the dependence of $E C_{\mathrm{a}}$ on $\theta$ and the electrical conductivity of the soil water $E C_{w}$, these two measurements promise the ability to determine $E C_{w}$ from a TDR wave-form. For conditions under which the soil water chemistry is dominated by a single electrolytic solute, $E C_{w}$ will be linearly related to the solute concentration, allowing for solute concentration measurement with TDR. Techniques for measuring the parameters of the transport models based on TDR have recently become widespread. Such techniques can be used to measure the mass flux of solute past the ends of TDR probes and characterize the probability 
density function of solute travel time. The procedure may be applied to a conservative tracer under steady state leaching condition, provided that a solute pulse causing a measurable reduction in the TDR signal is added to soil surface.

As shown by various authors (Dalton and van Genuchten, [8]; Nadler et al., [9]; Kachanoski et al., [1]) the resident solute concentration $C_{\mathrm{r}}\left(\mathrm{ML}^{-3}\right)$ in the soil may be determined from estimates of bulk soil electrical conductivity $E C_{\mathrm{a}}\left(\mathrm{dSm}^{-1}\right)$, conducted by means of TDR.

In general, in a porous medium under conditions of steady flow, there are the following relations between the resident concentration $C_{\mathrm{r}}$, bulk electrical conductivity $E C_{\mathrm{a}}$ and impedance $Z_{\infty}(\mathrm{ohm})$, hereafter referred as $Z$, of the transmission line measured by TDR:

$$
\begin{aligned}
& C_{r}=a+b E C_{a} \\
& E C_{a}=\frac{K}{Z-Z_{c}}
\end{aligned}
$$

in which $a$ and $b$ are calibration constants, $K\left(\mathrm{~L}^{-1}\right)$ is the cell constant of the TDR probe and $Z_{\mathrm{c}}$ is the impedance associated to the cable, the connector and the tester (Topp et al., [5]).

Eqn. (1) holds for variable soil salinity levels ranging between 0 and $50 \mathrm{dSm}^{-1}$ and for different values of $\theta$ (Ward et al., [10]), even if at higher $\theta$ values some nonlinearity was observed for low concentrations. In fact, $E C_{\mathrm{a}}$ is linearly related to $E C_{\mathrm{w}}$ for a range between 1 and $20 \mathrm{dSm}^{-1}$ (Rhoades et al., [11]). As was also shown, $E C_{\mathrm{w}}$ is linearly related to pore water solute concentration $C_{\mathrm{r}}$ for a similar range. Thus the $E C_{\mathrm{a}}-C_{\mathrm{r}}$ relationship is approximately linear for this electrical conductivity range (Kachanosky et al., [1]). The nonlinearity observed is held to be affected by the clay content in the soils and by $\mathrm{Na}^{+}$saturation, water content and probe size. Thus, the relation at low $E C_{\mathrm{w}}$ values, with $\theta$ constant, is soilspecific and should be estimated.

Direct calibration of the TDR probe in different solutions with known $E C_{w}$ (0-12 $\left.\mathrm{dSm}^{-1}\right)$ can be used to calculate $Z_{\mathrm{c}}$ and $K$ (Heimovaara, [12]). For salinity levels less than about $3 \mathrm{dSm}^{-1} Z_{\mathrm{c}}<<Z_{\infty}$ (Mallants et al., [13]).

The relative concentration of the solute resident in the volume sampled by a TDR probe of length $L$ is given by:

$$
C_{r}^{*}(L, t)=\frac{C(t)-C_{0}}{C_{0}-C_{i}}
$$

equivalent to:

$$
M^{*}(L, t)=\frac{M(t)}{M_{0}}
$$

where $C_{0}$ is the concentration of the applied solution, $C_{\mathrm{i}}$ is the original concentration in the porous medium, $C(t)$ is the concentration for greater time values, $M^{*}$ is the relative solute mass, $M(t)\left(\mathrm{ML}^{-2}\right)$ is the total mass of solute between the pair of rods at any time $t$ and $M_{0}$ is the total mass of solute contained between the rods from soil surface to the bottom end of the probe. 
By substituting eqn. (1) in eqn. (3) and by using eqn. (2) to eliminate the constants, the following is obtained (Kachanoski et al., [1]):

$$
M^{*}(L, t)=C_{r}^{*}(L, t)=\frac{Z^{-1}(t)-Z_{i}^{-1}}{Z_{0}^{-1}-Z_{i}^{-1}}
$$

where $Z_{\mathrm{i}}$ is the initial impedance before applying the solution, $Z_{0}$ is the impedance associated with the applied concentration $C_{0}$, equation (5) shows that, with $\theta$ constant, the distribution $C^{*}(L, t)$ may be obtained from measurements of impedance $Z(L, t)$ if a particular value of impedance $Z_{0}$ can be related to a known concentration such as the concentration of the inlet solution $C_{0}$.

In a displacement experiment with a vertical TDR probe configuration, assuming that the TDR probe extends from the surface to a depth $L$, addition of a solute pulse with total specific mass $M_{0}$ at time $t_{0}$ immediately leads to a drop in impedance $Z$. When the finite solute pulse is wholly located in the volume observed by the TDR probe, the impedance will have a constant minimum value $Z_{0}$ associated to $M_{0}$. However, as the solute moves below the bottom end of the TDR probe, the impedance will tend to gradually increase and reach its initial value $Z_{i}$ when all the solute is past the probe.

It is currently possible to obtain $Z$ values directly from the display of a Tektronix (mod.1502C) cable tester. In this study, the readings were conducted at the intersection of the tester display cursor with the trace of the reflected signal when the width of the latter appeared stabilised. According to Nadler et al. [9], the problem of multiple reflections may be overcome by reading the impedance at a late time $(t \rightarrow \infty)$ along the signal when all multiple reflections are suppressed. The differences in $Z$ are used in the calibration process to obtain relative mass distribution (Kachanosky et al., [1]) from which in turn solute flux concentration at $z=L$ can be derived (Elrich et al., [14]).

By deriving eqn. (4) as regards time, the flow density relative to the solute may be obtained:

$$
f(L, t)=\frac{1}{Z_{0}^{-1}-Z_{i}^{-1}} \frac{\partial}{\partial t}[Z(t)]^{-1}
$$

Equation (6), equivalent to the probability density function ( $p d f)$ of solute travel time, may be considered as a characteristic of the volume of solute transport and may thus be assumed directly as a transfer function in the transfer function model TFM (Jury et al., [15]).

\subsection{Miscible transport experiments}

Methodology illustrated above was verified with reference to three undisturbed soil samples $150 \mathrm{~mm}$ in diameter and $170 \mathrm{~mm}$ in length from different sites of southern Italy. The samples were collected by means of steel samplers guided by an appropriate hydraulic device, ensuring the simultaneous removal of surrounding material so as to reduce alterations and compaction of the samples.

Samples were identified as sandA, clay and $s a n d B$ respectively. Their main physical characteristics as determined in the laboratory, together with some 
pedological characteristics of the horizons and the sampling site, are reported in Table 1 (Comegna et al. [4]).

In the laboratory the samples were saturated slowly from the bottom. Subsequently, the saturated hydraulic conductivity $K_{\mathrm{s}}$ was determined by the constant head method.

Table 1: $\quad$ Physical characteristics of the soils examined.

\begin{tabular}{|c|c|c|c|c|c|c|}
\hline $\begin{array}{c}\text { Texture } \\
\text { (ISSS) }\end{array}$ & $\begin{array}{c}\text { Sand } \\
(\%)\end{array}$ & $\begin{array}{c}\text { Silt } \\
(\%)\end{array}$ & $\begin{array}{c}\text { Clay } \\
(\%)\end{array}$ & $\begin{array}{c}\text { Bulk } \\
\text { density } \\
\left(\mathrm{g} / \mathrm{cm}^{3}\right)\end{array}$ & $\begin{array}{c}\text { Organic } \\
\text { matter } \\
(\%)\end{array}$ & $\begin{array}{c}\text { Pedological } \\
\text { classification }\end{array}$ \\
\hline $\begin{array}{c}\text { Sandy } \\
\text { (sand A) }\end{array}$ & 88.0 & 6.0 & 6.0 & 1.297 & 3.1 & $\begin{array}{c}\text { Horizon A: } \\
\text { crumb } \\
\text { structure; } \\
\text { entisol }\end{array}$ \\
\hline $\begin{array}{c}\text { Clayey sand } \\
\text { (clay) }\end{array}$ & 39.72 & 25.43 & 34.85 & 1.240 & 1.9 & $\begin{array}{c}\text { Horizon A: } \\
\text { polyedric- } \\
\text { subang. } \\
\text { structure; } \\
\text { vertisol }\end{array}$ \\
\hline $\begin{array}{c}\text { Sandy } \\
\text { (sand B) }\end{array}$ & 80.0 & 12.0 & 8.0 & 1.285 & 2.1 & $\begin{array}{c}\text { Horizon A: } \\
\text { granular } \\
\text { structure; } \\
\text { andosol }\end{array}$ \\
\hline
\end{tabular}

Subsequently, in a thermoregulated chamber $\left(20 \pm 0.5^{\circ} \mathrm{C}\right)$, the samples to be characterised were subjected to very simple one-dimensional flow processes with a specifically constructed experimental leaching configuration. The leaching unit shown in figure 1 consists essentially of a soil column, a rain simulator, a vacuum unit and allows saturated and unsaturated flow experiments to be conducted.

The bottom end cap of the column supports a nylon cloth of $25 \mu$ mesh-wire with a bubbling pressure of $\approx 2.5 \mathrm{kPa}$. A bubble tower with a movable air entry tube allows the pressure potential $h$, of water to be imposed at the bottom of the sample. The column is then equipped with a vertically installed bifilary TDR probe for measuring the water content $\theta$ and impedance $Z$, and with two tensiometers for measuring the water potential $h$.

The laboratory-built TDR probe consists of two $5 \mathrm{~mm}$ diameter steel rods, 50 $\mathrm{mm}$ apart, $150 \mathrm{~mm}$ long extending from a perspex head enclosing a 1:1 matching ferrite balun (Spaans and Baker, [6]) and is connected to the measuring device by a $2 \mathrm{~m}$-long coaxial cable (RG $58 \mathrm{U}$ ) with a characteristic impedance of $50 \Omega$. Porous fritted glass plate tensiometers $(10 \mathrm{~mm}$ in diameter and with a bubbling pressure of $\approx 50 \mathrm{kPa}$ ) are inserted horizontally $5 \mathrm{~cm}$ and $15 \mathrm{~cm}$ down the soil column and are connected to pressure transducers. The leaching unit is completed by other basic components, namely: i) a Tektronix Mod. 1502C metallic TDR cable tester equipped with an RS 232 interface (Tektronix Inc.); ii) a personal computer for control, acquisition and data analysis; iii) a 50-needle (id. $0.6 \mathrm{~mm}$ ) rainfall simulator; iv) a peristaltic pump serving the simulator; v) an automatic fraction collector to collect the effluent in small fractions $(10-15 \mathrm{cc})$. 


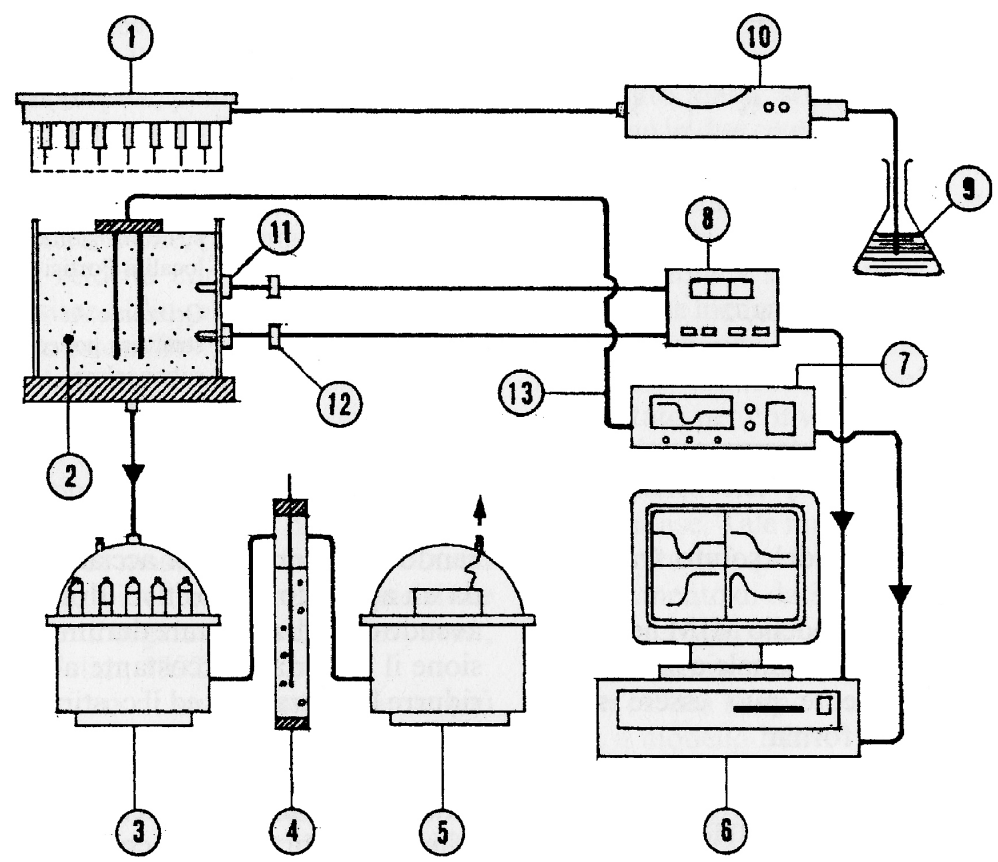

Figure 1: Schematic diagram of the laboratory apparatus showing: (1) rain simulator, (2) soil monolith, (3) fraction sampler, (4) Mariotte vessel, (5) vacuum pump, (6) computer, (7) TDR tester, (8) multiplexer, (9) tracer vessel, (10) peristaltic pump, (11) tensiometer, (12) pressure transducer, (13) coaxial cable.

Each sample thus underwent a preliminary conditioning phase by feeding, with a steady water flux density, the flow system with a $0.01 \mathrm{~N} \mathrm{CaSO}_{4}$ solution until the steady flow and initial chloride concentration $C_{\mathrm{i}}=0$ were reached. Contemporaneous measurements of $\theta, h$ and $q$ allowed us to determine the onset of steady-state. At steady state, the solute was applied in a different way depending on the saturation degree during the experiments.

Concerning saturated experiments, the input of water was interrupted and a pulse of $\mathrm{KCl}$ of total specific mass $M^{*}$ corresponding to $26.9 \mathrm{~g} / \mathrm{cm}^{2}$ of chloride, obtained by dissolving $1 \mathrm{~g}$ of $\mathrm{KCl}$ in $50 \mathrm{cc}$ of water, was applied at the top of the sample for a variable period of time $\mathrm{t}_{0}$ from 4 to $7 \mathrm{~min}$. The chloride pulse, which had in the meantime completely penetrated the upper layers of the sample, was shifted by re-feeding the sample with the $\mathrm{CaSO}_{4}$ solution.

During leaching tests the leachate was periodically collected. The chloride ion concentration at the outlet boundary was determined by titrating $10 \mathrm{~mL}$ of aliquot against standard $\mathrm{AgNO}_{3}$ using $5 \% \mathrm{~K}_{2} \mathrm{CrO}_{4}$ as an indicator (=reference method) (Richards, [16]). 
The $Z(t)$ time series were obtained and used to estimate by eqn. (5) the relative solute mass $M^{*}(L, t)$ localized between the surface and end of the TDR probe of length $L$.

\section{Interpretative models of chemical transport}

One-dimensional steady flow of an inert solute in homogeneous and isotropic porous media may be described by the classical convection-dispersion differential equation $(\mathrm{CD})$ :

$$
R \frac{\partial C}{\partial t}=D \frac{\partial^{2} C}{\partial x^{2}}-v \frac{\partial C}{\partial x}
$$

where $C\left(\mathrm{ML}^{-3}\right)$ is the solution concentration, $v=q / \theta\left(\mathrm{LT}^{-1}\right)$ is the average pore water velocity, $q\left(\mathrm{LT}^{-1}\right)$ is the Darcy velocity, $\theta$ the volumetric water content, $D$ $\left(\mathrm{L}^{2} \mathrm{~T}^{-1}\right)$ the coefficient of hydrodynamic dispersion, $R$ the retardation coefficient accounting for equilibrium linear sorption processes, $x(\mathrm{~L})$ is the distance in the direction of the flow and $t(\mathrm{~T})$ is time. Instantaneous concentrations $C$ provided by TDR electrical resistance measurement are defined as the mass of solute per unit of soil volume $C_{r}$.

The hydrodynamic dispersion coefficient $D$ has commonly been expressed as $D=\lambda v_{0}{ }^{n}+D_{0}$, where $\lambda$ and $n$ are constants. $\lambda$ is a characteristic property of the porous medium usually referred as dispersivity and $n$ is commonly taken as a value between 1-2 (Kutilek and Nielsen, [2]).

On the basis of heuristic reasoning supplied by Jury et al. [3], equation (7) describes simply dispersion on a macroscopic scale in a homogeneous porous medium, hypothesizes implicitly that there is a Gaussian distribution of velocity in the flow domain and typically supplies a signal which may be modelled by a dispersion coefficient $D$ in which all approximation errors are propagated.

During experiments some BTCs could show the establishment of a nonequilibrium indicated by tailing and early appearance of tracer in the effluent, mechanisms that generally cannot be described by analytical solutions supplied by equation (7). The mobile-immobile convection-dispersion concept (MIM) (van Genuchten and Wierenga, [17]; Gaudet et al., [18]) can be used for solute transport in such soils. In this conceptual model the porous medium is divided into two transport regions (mobile and immobile). Convective transport is assumed to occur only in the so-called mobile region, which is a fraction $\beta$, of the water filled pore space.

$$
\begin{aligned}
\theta_{m} \frac{\partial C_{m}}{\partial t}+\theta_{i m} \frac{\partial C_{i m}}{\partial t} & =\theta_{m} D_{m} \frac{\partial^{2} C_{m}}{\partial x^{2}}-\theta_{m} v_{m} \frac{\partial C_{i m}}{\partial x} \\
\theta_{i m} \frac{\partial C_{i m}}{\partial t} & =\alpha\left(C_{m}-C_{i m}\right)
\end{aligned}
$$


Chemical exchange between the two regions is assumed to be a diffusive process and is macroscopically described by a first-order kinetic, according to a difference of concentration, with a rate coefficient $\alpha$.
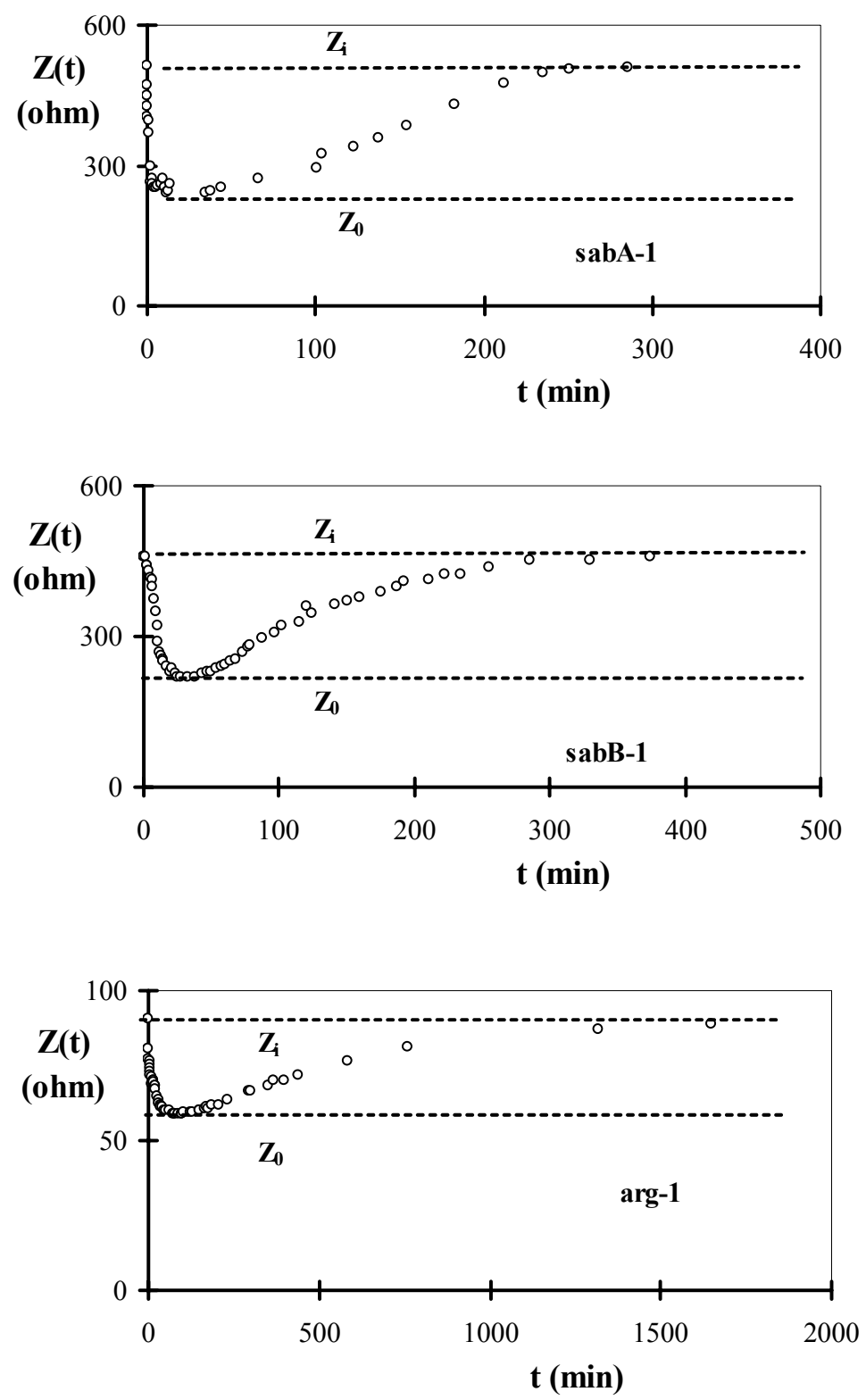

Figure 2: $\quad Z(t)$ values measured by TDR. 


\section{$4 \quad$ Results and discussion}

Overall, seven tests were carried out on sandA sandy soil, eight on $\operatorname{sandB}$ sandy soil and two on clay clayey soil. In order to illustrate the characteristic pattern of the electric impedence of transmission line in the soil, figure 2 reports as an example the time series of $Z(t)$ recorded during experiment sandA-1, sandB-1 and clay- 1 conducted on respective columns at lclayest $v_{0}$ value and at complete saturation.

It may be observed from the figures that the initial impedance $Z_{\mathrm{i}}$ varies from $520 \mathrm{ohm}$ for sandA-1 to $460 \mathrm{ohm}$ for sandB-1 to $93 \mathrm{ohm}$ for clay-1. Such differences may be attributed to the different chemical and physical characteristics of the soils examined.

Moreover, after application of the solute, significant variations are noted in impedance values, which in a short space of time record minimum $Z_{0}$ values of $250 \mathrm{ohm}, 220 \mathrm{ohm}$ and $58 \mathrm{ohm}$ respectively for the three different soils. Finally, as shown by the times required to return to the $Z_{i}$ values, the different physical and chemical characteristics of the soils also resulted in different time dynamics of solute transport.

The obtained values of $Z_{i}, Z_{0}$ and $Z(t)$ were re-used to plot the BTCs, $C(t)$, normalized between 0 and 1 . For all the soils a straightforward comparison between TDR derived concentrations and those measured with the reference method may be established in figure 3 which represents the pertaining $p d f s$.

For $s a n d A-1$ and $s a n d B-1$, the pdfs measured with TDR and the reference method are comparable to a slightly asymmetric bell-shaped curve. The results compare well, showing differences only for $\mathrm{t}>80 \mathrm{~min}\left(\mathrm{r}^{2}=0.960\right)$ and for $\mathrm{t}>60 \mathrm{~min}$ $\left(r^{2}=0.974\right)$. By contrast, for vertisol, the $p d f s$ are typically asymmetric with accentuated tails (figure 4). A differentiation of the $p d f s$ is noted, with clear deviations from the reference values, for times $\mathrm{t}<120 \mathrm{~min}\left(\mathrm{r}^{2}=0.882\right)$. In this case, the $p d f$ measured with TDR leads to a slight overestimate of the time corresponding to the peak concentration and thus to a slight underestimate of the modal velocity of the solute.

Just as an example of results obtained under unsaturated conditions, figure 4 shows the diagram of $Z(t)$ values for the experiment sandA-2, along with the comparison between derived concentration values and those measured by the reference method.

The very slight discrepancies between the compared experimental BTCs emphasise the good agreement of the experimental devices and techniques adopted. For all the other experiments conducted, not reported here for the sake of brevity, it was observed that the initial impedence $Z_{i}$, corresponding to concentration $C_{i}$, varies within a range between 700 and $519 \mathrm{ohm}$. Such differences may generally be attributed to the variability in bulk soil electrical conductivity, the different water and clay contents of soils. After application of the solute, significant variations in impedence were noted which, with different time laws, showed minimum $Z_{0}$ values, corresponding to the applied concentration $C_{0}$, between $38-83 \mathrm{ohm}$. 

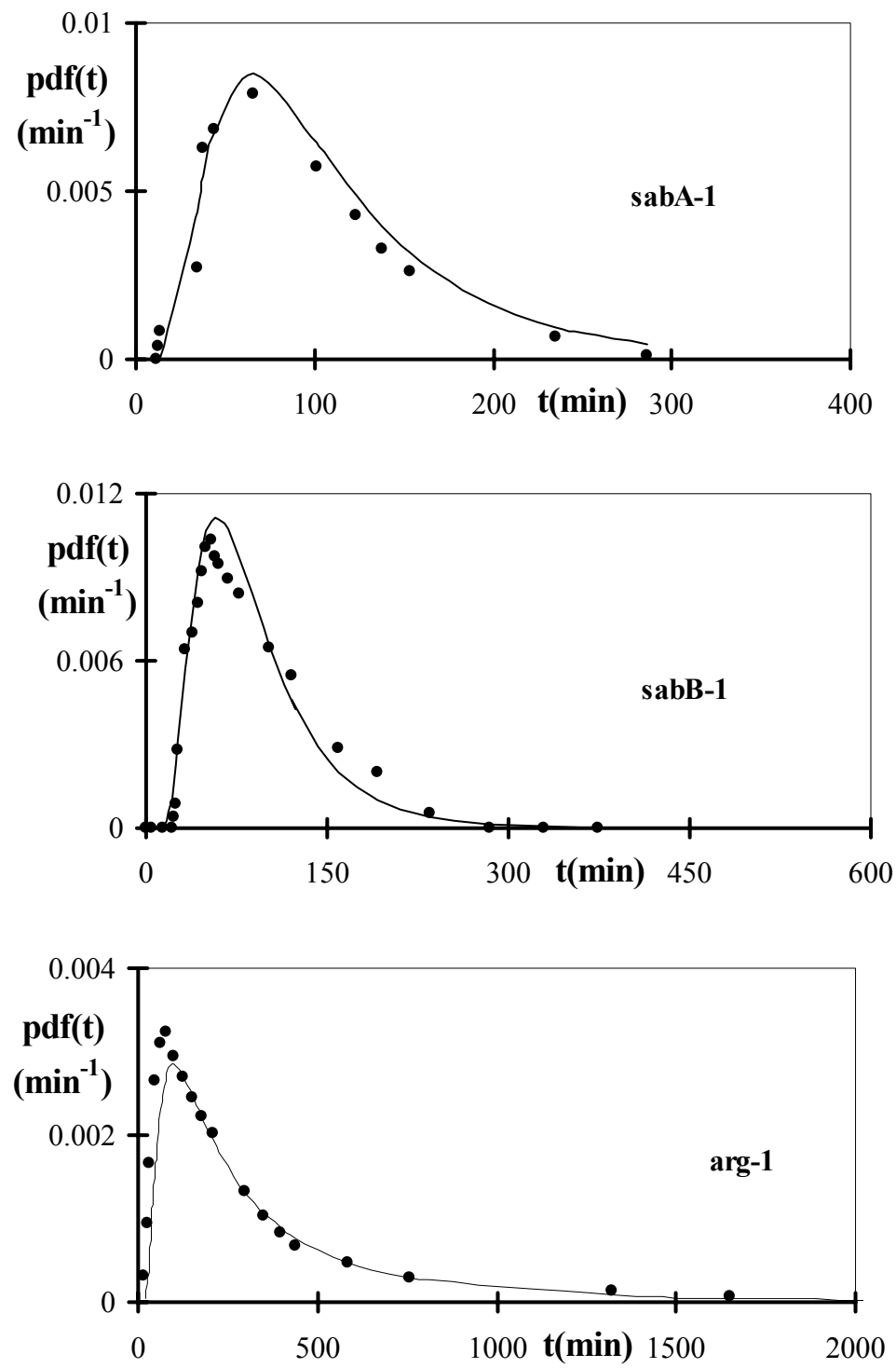

Figure 3: Comparison between $\operatorname{pdf}(\mathrm{t})$ obtained by $\operatorname{TDR}(-)$ and $\operatorname{pdf}(\mathrm{t})$ obtained by the reference method $(\bullet)$.

The experimental BTCs were first modeled with the CD model (equation (6)). The model parameters were determined using the CXTFIT program (Toride et al., [19]) which minimizes the sum of the squares of the residuals between the measured values and those calculated with an analytical solution of type A1 
proposed by van Genuchten and Wierenga [17]. So as to reduce the number of iterations, the actual velocity $v_{0}$ was set equal to the $q / \theta$ ratio and initial estimates used of parameters $R$ and $D$ were reasonably close to optimal values, obtained with the deterministic methods proposed by Fried and Coubernous [20].

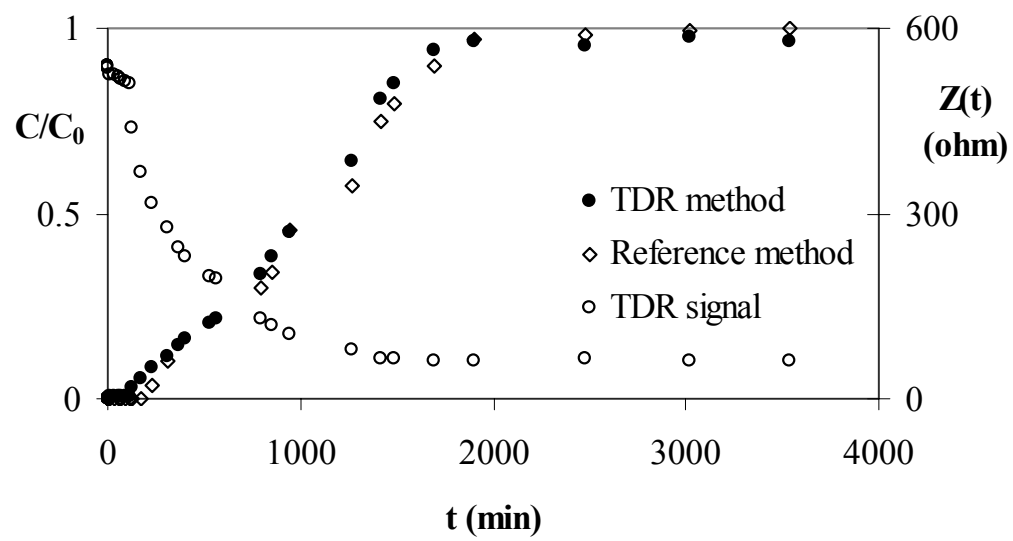

Figure 4: $\quad$ Measured impedance load $Z(t)$ (sandA-2 experiment) from the cable tester $(0) ; C(x, t)$ values measured with $\operatorname{TDR}(\bullet)$ and reference method $(\diamond)$.

Table 2: $\quad$ Parameters obtained by fitting the CD model to the experimental BTCs. $R^{*}$ and $D^{*}$ parameters starting values obtained by using the methods of van Genuchten and Wierenga (1976) and Fried and Combarnous (1971), respectively.

\begin{tabular}{|l|c|c|c|}
\hline \multicolumn{1}{|c|}{ Parameters } & Entisol & Vertisol & Andosol \\
\hline $\mathrm{q}\left(\mathrm{cm} \mathrm{min}^{-1}\right)$ & 0.0715 & 0.0189 & 0.0732 \\
\hline $\mathrm{Ks}\left(\mathrm{cm} \mathrm{min}^{-1}\right)$ & 0.0640 & 0.0343 & 0.0343 \\
\hline$\theta_{\mathrm{s}}$ & 0.410 & 0.489 & 0.370 \\
\hline $\mathrm{v}\left(\mathrm{cm} \mathrm{min}{ }^{-1}\right)$ & 0.174 & 0.039 & 0.198 \\
\hline $\mathrm{R}^{*}$ & 1.270 & 1.092 & 1.187 \\
\hline $\mathrm{R}$ & 1.280 & 1.070 & 1.201 \\
\hline $\mathrm{D}^{*}\left(\mathrm{~cm}^{2} \mathrm{~min}^{-1}\right)$ & 0.418 & 0.184 & 0.658 \\
\hline $\mathrm{D}\left(\mathrm{cm}^{2} \mathrm{~min}^{-1}\right)$ & 0.275 & 0.146 & 0.451 \\
\hline $\mathrm{P}$ & 9.512 & 3.962 & 6.580 \\
\hline$\lambda(\mathrm{cm})$ & 1.577 & 3.786 & 2.280 \\
\hline $\mathrm{r}^{2}$ & 0.980 & 0.997 & 0.988 \\
\hline $\mathrm{MSE}$ & 0.024 & 0.012 & 0.027 \\
\hline
\end{tabular}

Seemingly a good agreement between calculated and measured BTCs was achieved, as confirmed by the high $\mathrm{r}^{2}$ obtained also for experiments not shown here. 
Though the shape of some breakthrough curves and pertaining parameters seemed indicate the establishment of a non-equilibrium, especially on columns labeled as sandA and clay, curve-fitting MIM model to experimental breakthrough produced always unimproved fittings and unreliable parameter estimates as compared to the $\mathrm{CD}$ results. Thus, deducting physical meanings from the uncertainly parameters obtained by MIM model would have been hazardous and probably meaningless. Accordingly, the obtained MIM parameters are not shown herein and all the following analyses were performed considering the $\mathrm{CD}$ as the appropriate model.

\subsection{Sensitivity of hydrodynamic dispersion to experimental errors}

Impedance $Z$, and average pore water velocity $v$, are the experimental parameters mainly involved in the hydrodynamic dispersion estimation. Any error in their measurement propagates on the dispersion coefficient in a way which can be studied through simulations.

Starting from an experimental curve for which the closest fitting was obtained, the sensitivity of hydrodynamic dispersion to errors in velocity determination was investigated by applying fictitious variations to its experimental value in the range $-10 \%-10 \%$. Velocity was first fixed while optimizing both dispersion and retardation coefficients. In this case a different hydrodynamic dispersion was obtained depending on the starting velocity value.

To the contrary, a constant dispersion was obtained by optimizing both dispersion and velocity parameters, thus demonstrating the timeliness of optimizing velocity when unstable water content and/or inflow were hypothesized or observed during experiments. Results referring to these trials are observable in figure $5 \mathrm{a}$ and $5 \mathrm{~b}$.

Concerning the influence of impedance determinations on hydrodynamic dispersion, new fictitious experimental curves were calculated by perturbing the experimental $M^{*}(L, t)$ obtained from equation (5) through a random error which was calculated according to the following equation:

$$
M_{p}^{*}(L, t)=M^{*}(L, t)[1+2 f(F-0.5)]
$$

where $M_{p}{ }^{*}(L, t)$ is the relative solute mass which is assumed to be subject to random measurement error, $F$ is a random number between 0 and 1 .

Finally, $f$ is the relative error to which values of $0.05,0.10$ and 0.15 are assigned in order to simulate errors of 5, 10 and 15\% respectively. Parameter estimation analysis was carried out on the fictitious realizations of data using values shown in Table 2 for the experiment sandA-2 as starting values for transport parameters.

Graphical results are illustrated in figure 6. An appreciable sensitivity to measurement errors was observed just when the $15 \%$ error was considered, with a large deviation of $D$ from its "true" value. Nevertheless, when 5 and $10 \%$ errors were applied, the observed deviations were limited to 1.1 and $2 \%$ respectively. 


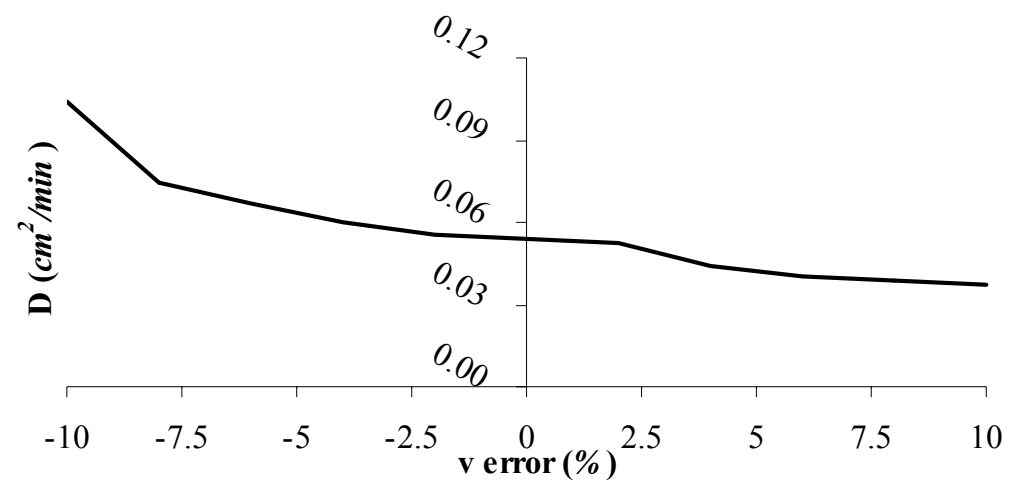

(a)

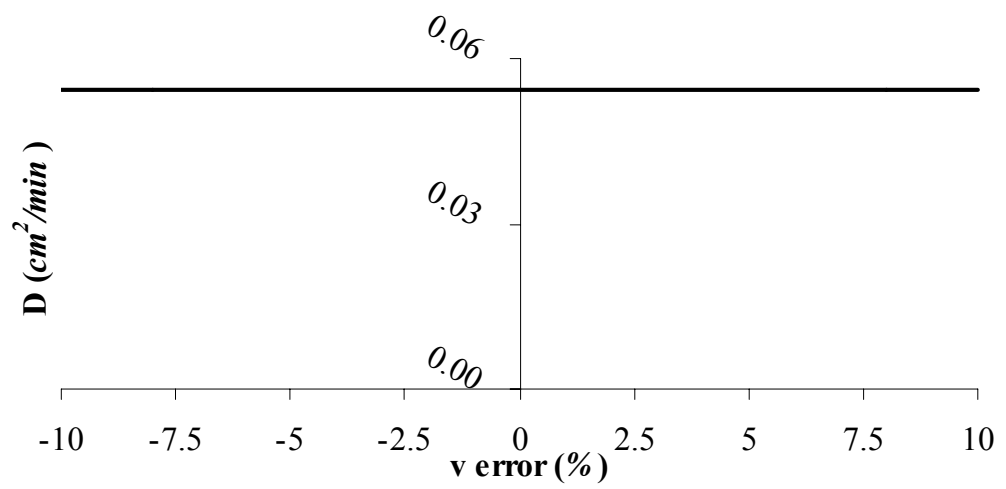

(b)

Figure 5: (a) Hydrodynamic dispersion coefficient as obtained by applying measurement errors to pore water velocity, keeping $v$ fixed during the fitting; (b) Hydrodynamic dispersion coefficient as obtained by applying measurement errors to pore water velocity $v$ is optimized during the fitting.

\section{Conclusions}

TDR technology, though already used in the field of soil physics and soil hydrology to measure water content in natural porous media, has only recently begun to be used to estimate chemical transport parameters in soil.

The illustrated methodology, which considers laboratory tests on undisturbed soil samples, permits considerable reductions in the number of measurements, while supplying results which are at least as accurate as those obtainable from long-established conventional techniques. 


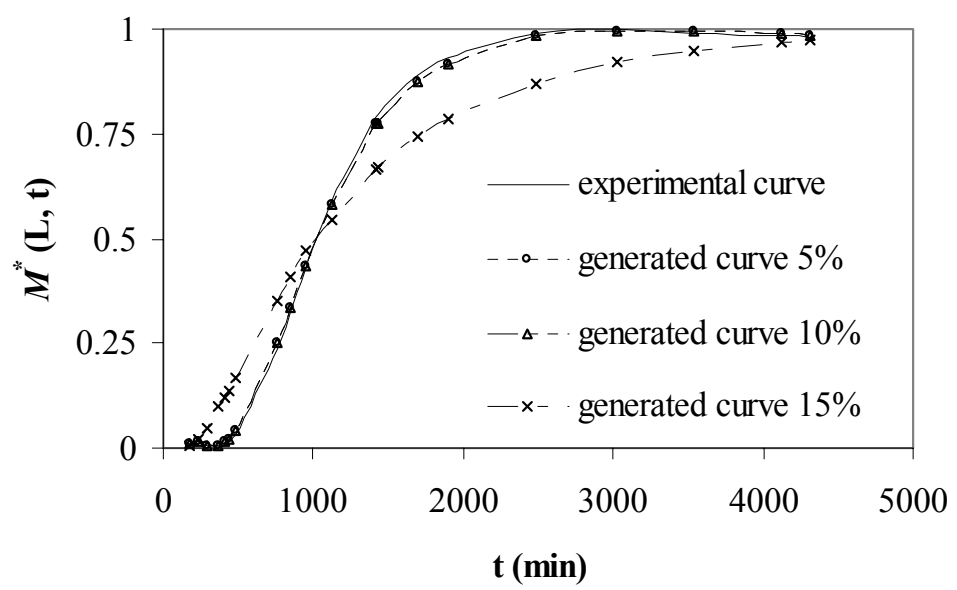

Figure 6: Effect of errors in the determination of $M^{*}(\mathrm{~L}, \mathrm{t})$ on the fitted curve.

Tested on soils with clearly different physical characteristics, the method allows reliable definition of transport parameters in steady flow conditions and for water content near saturation.

With regard to sandy soils, the two-parameter CD model supplied optimal estimates of concentrations, $C(x, t)$. As regards clayey soil, the use of the CD equation is not necessarily ruled out although a lower $r^{2}$ value was calculated. To reduce the greater discrepancies observed between the calculated curves and the measured data, more sophisticated versions of the CD model must be used, such as the CD-MIM model, which is known to compartmentalise the liquid phase into two domains, mobile and immobile. Nevertheless, CD appears a very reliable model which is appropriate for mechanistic of the solute transport process. Applying the technique to field trials may be extremely useful. In order to obtain sufficiently precise estimates of transport parameters for applications, techniques will have to be set up which take account of soil heterogeneity and the uncertainty of boundary conditions. Further and more extensive verification of the methodology is, however, necessary both in the laboratory and in the field, as well as on different soils.

\section{References}

[1] Kachanosky R.G., E. Pringle and A. Ward, Field measurement of solute travel time using time domain reflectometry. Soil Sci. Soc. Am. J. 56:4752, 1992.

[2] Kutilek M. and D.R. Nielsen, Soil Hydrology. Catena. Verlag. Geoscience Publisher, pp.370, 1994.

[3] Jury W. A., W. R. Gardner and W. H. Gardner, Soil Physics. Wiley, NY, 5th. ed., pp.328, 1991. 
[4] Comegna V., A. Coppola and A. Sommella, Nonreactive solute transport in variously structured soil materials as determined by laboratory-based time domain reflectometry (TDR). Geoderma, 92:167-184, 1999.

[5] Topp G.C., J.L. Davis and A.P. Annan, Electromagnetic determination of soil water content: measurements in coaxial transmission lines. Water Resour. Res. 16:574-582, 1980.

[6] Spaans E.J.A. and J. M. Baker, Simple baluns in parallel probes for time domain reflectometry. Soil Sci. Soc. Am. J. 57:668-673, 1993.

[7] Dalton F.N., Herkelrath W.N., Rawlins D.S., Rhoades J.D., Time Domain Reflectometry: simultaneous measurements of soil water content and electrical conductivity with a single probe. Science 224, 989-990, 1984.

[8] Dalton F.N. and van Genuchten M.Th., The time domain reflectometry method for measuring soil water content and salinity. Geoderma, 38: 237250, 1986.

[9] Nadler A., Dasberg, S. and Lapid, I., Water content and electrical conductivity determination of layered soil profiles using time domain reflectometry. Soil Sci. Soc. Am. J., 55: 938-943, 1991.

[10] Ward A.L., Kachanoski R.C. and Elrick D.E., Laboratory measurement of solute transport using time domain reflectometry. Soil Sci. Soc. Am. J., 58, 1031-1039, 1994.

[11] Rhoades J.D., N.A. Manteghi, P.J. Shouse and W.J. Alves, Soil electrical conductivity and soil salinity: new formulations and calibrations. Soil Sci. Soc. Am. J., 52:433-439, 1989.

[12] Heimovaara, T.J., Time domain reflectometry in soil science: Theoretical backgrounds. Measurements and models. Ph. D. diss. Univ. of Amsterdam, 1993.

[13] Mallants D., M. Vanclooster, N. Toride, J. Vanderborght, M.Th. van Genuchten and J. Feyen, Comparison of three methods to calibrate TDR for monitoring solute movement in undisturbed soil. Soil Sci. Soc. Am. J., 60:747-754, 1996.

[14] Elrick D.E., R.G. Kachanoski, E.A. Pringle and A.L. Ward, Parameter estimates of field solute transport models based on time domain reflectometry measurements. Soil Sci. Soc. Am. J., 56:1663-1666, 1992.

[15] Jury W.A., Stalzy L.H. and Shouse D., A field test of the transfer function model for predicting solute transport. Water Resour. Res., 18: 369-375, 1982.

[16] Richards L.A., Diagnosis and improvement of saline and alkali soils. USDA Handbook, 60. U.S. Government Printing Office, Washington D.C., 1968.

[17] van Genuchten M.Th. and P.J. Wierenga, Mass transfer studies in sorbing porous media: I. Analytical solutions. Soil Sci. Soc. Am. J. 40:473-480, 1976.

[18] Gaudet J.P., H. Jegat G. Vachaud and P.J. Wierenga, Solute transfer, with exchange between mobile and stagnant water, through unsaturated sand. Soil Sci. Soc. Am. J. 41:665-670, 1977. 
100 Sustainable Irrigation Management, Technologies and Policies

[19] Toride N, F.J. Leij and M.Th. van Genuchten, The CXTFIT Code for Estimating Transport Parameters from Laboratory or Field Tracer Experiments. Version 2.0, Research Report No. 137, U.S. Salinity Laboratory Agricultural Research Service. U.S. Department of Agriculture, Riverside, California, 1995.

[20] Fried J.J. and M.H. Coubernous, Dispersion in porous media. Adv. Hydroscience 7:169-282, 1971. 